\title{
Evaluación de la influencia de los gases de combustión en procesos de inyección continua de vapor utilizando generadores de vapor en fondo
}

\author{
Evaluation of the influence of combustion gases in \\ steamflooding process using downhole steam generators
}

\section{Avaliação da influência dos gases de combustão nos processos de injeção de vapor usando geradores de vapor de poço}

\author{
Ana Paula Villaquirán Vargas*; Astrid Xiomara Rodríguez Castelblanco; Samuel Fernando Muñoz Navarro. \\ Grupo de Investigación Recobro Mejorado. Escuela de Ingeniería de Petróleos. Universidad Industrial de \\ Santander (UIS), Cra. 27 Calle 9, Bucaramanga, Colombia. \\ *ana.paula.villaquiran.vargas@gmail.com o grm@uis.edu.co
}

Fecha Recepción: 21 de abril de 2017 Fecha Aceptación: 17 de agosto de 2017

\begin{abstract}
Resumen
Son numerosas las teorías que circundan los generadores de vapor en fondo, algunas son alentadoras y otras tratan de opacar el desarrollo de la tecnología; en el presente trabajo se analizaron los tipos de generadores de vapor en fondo, así como su funcionamiento básico, se indicaron ventajas y desventajas de las herramientas en fondo, comparándolas con las ubicadas en superficie. Con base en la literatura, se realizaron hipótesis sobre el efecto de los gases producto de la combustión y posteriormente, se construyó un modelo conceptual de simulación para comprobar o refutar los planteamientos anteriores. A pesar de que el gas no condensable que se encontraba en mayor proporción, con combustible aire, era el nitrógeno, distinguido por poseer altas presiones mínimas de miscibilidad, se pudo evidenciar que el efecto de los gases emitidos al yacimiento por el generador en fondo, era de tipo miscible, incrementando el factor de recobro en aproximadamente un 30\% en comparación con un generador convencional. Los buenos resultados obtenidos son un índice de que es necesario continuar con las investigaciones y progreso de los generadores de vapor en fondo, hasta encontrar la herramienta y la fórmula de uso adecuada.
\end{abstract}

Palabras clave: inyección de vapor, generadores de vapor en fondo, DSG, modelo conceptual, simulación de yacimientos, gases de combustión.

\begin{abstract}
There are numerous theories around the downhole steam generators, some are encouraging and others try to overshadow the development of technology, this paper has a review of the types of downhole steam generators and its basic functioning, also is presented some advantages and disadvantages of the technique comparing them with those located on the surface. Based on the literature, hypotheses about the effect of the combustion product gases were performed and subsequently a conceptual simulation model was built to prove or disprove the above approaches. It was determined that the effect of the exhaust gases is characterized in the reservoir for an miscible process. Good results are an indication that it is necessary to continue researching and making efforts, to find the appropriate tool and its best formulation.
\end{abstract}

Keywords: steam injection, downhole steam generator, DSG, conceptual model, reservoir simulation, combustion gases.

Cita: Villaquirán Vargas AP, Rodríguez Castelblanco AX, Muñóz Navarro SF. Evaluación de la influencia de los gases de combustión en procesos de inyección continua de vapor utilizando generadores de vapor en fondo. rev.ion. 2017;30(2):65-77. 


\section{Resumo}

Existem muitas teorias em torno dos geradores de vapor de fundo de poço, algumas são alentadoras, mas outras tentam obstruir o desenvolvimento desta tecnologia. Neste trabalho, foi realizada uma revisão bibliográfica dos tipos de geradores de vapor de fundo, bem como o seu funcionamento básico, indicando vantagens e desvantagens das ferramentas de fundo em comparação com as localizadas na superfície. Com base na literatura, foram desenvolvidas hipóteses sobre o efeito dos gases provenientes da combustão e, posteriormente, foi construído um modelo de simulação conceitual para verificar ou contradizer as abordagens anteriores. Determinou-se que o efeito dos gases produzidos pela combustão do gerador no reservatório está caraterizado pelo empuxo miscível, embora o gás não condensável que esteja em maior proporção, com combustível de ar, é o nitrogênio que se distingue por ter alta pressões mínimas de miscibilidade. Os bons resultados obtidos indicam que é necessário continuar com as investigações e o progresso dos geradores de vapor de fundo, até encontrar a ferramenta juntamente com a fórmula de uso apropriada.

Palavras-chave: steamflood, geradores de vapor de fundo de poço, modelo conceptual de simulação, simulação de reservatórios, gases de combustão.

\section{Introducción}

La inyección de vapor ha sido empleada en yacimientos de crudos pesados, los cuales son primordialmente someros. En la literatura datan distintas profundidades límite o máximas, para implementar de manera efectiva este método, la más conocida es de 3.000ft [1]. No obstante, es de vasto conocimiento la existencia de campos de crudo pesado con profundidades mayores que requieren de un proceso térmico que reduzca la viscosidad del aceite, como es el caso de Castilla, Quifa y Chichimene en Colombia [2], campos donde habrian altas pérdidas energéticas desde superficie hasta la cara del pozo si se implementara un método de recobro de este tipo con un generador convencional, razón por la cual se plantea el uso de generadores de vapor en fondo con el fin de reducir dichas pérdidas y poder producir el petróleo más fácilmente.

Los primeros generadores de vapor en fondo fueron creados en la década de los setenta [3]; sin embargo, no fueron diseñados para estar dentro del pozo, sino que simplemente se incorporaron a una herramienta existente, razón por la cual presentaron ciertos problemas como corrosión de las líneas y la herramienta en sí misma [4]. Con el tiempo se han perfeccionado los diseños, llegando a tener mejores y más precisas herramientas.

Dentro de los generadores de vapor en fondo se encuentran los generadores de combustión, los cuales presentan dos clasificaciones: Generadores de contacto indirecto y contacto directo. Son precisamente estos últimos los de mayor uso, y se caracterizan por su capacidad de inyectar los gases de combustión junto con el vapor generado.
El fin de la construcción de un modelo conceptual es el análisis y verificación del efecto de los gases de combustión mediante la simulación como herramienta de predicción.

Según la USGS (United States Geological Survey) más del $96 \%$ del petróleo pesado en el mundo proviene de profundidades superiores a 2.500 pies (Clasificación de Klemme); además, la recuperación térmica del petróleo pesado que reside bajo permafrost, como es el caso de Ugnu en Alaska, requiere un método de inyección de vapor que no afecte adversamente la integridad de la capa congelada [3]. Por esta razón se han desarrollado técnicas y tecnologías que puedan ser aplicadas en dichos rangos de profundidad y ambiente, como lo es DSG (por sus siglas en inglés downhole steam generators).

En 1978, Sandia National Laboratories recibió diferentes solicitudes para desarrollar un generador de vapor en fondo de pozo que pudiera dar solución a las grandes pérdidas de energía por el recorrido del vapor hasta formaciones profundas y los costos asociados a esto. Hart en 1982, demostró que la implementación del DSG se vuelve económicamente más atractiva en comparación con la generación de vapor en superficie que maneja tubería no aislada [5]. Así mismo, Capper en 2011 realizó un estudio de SAGD (por sus siglas en inglés Steam Assisted Gravity Drainage) con DSG, mostrando que los gases de combustión provenientes de un generador de fondo de contacto directo, tenían un efecto positivo en el proceso.

En 1980 se probó un DSG de contacto directo en el campo Kern River, Bakersfield, CA, obteniendo un aumento en la producción, una mejoría en el 
OSR (por sus siglas en inglés oil steam ratio) y una reducción en la contaminación del aire. No obstante, el tiempo de vida de la herramienta fue muy corto por problemas de corrosión [6]. Es por esto que un DSG requiere un diseño especial que asegure un desempeño confiable durante la operación, llevando a la industria petrolera a recurrir a desarrollos tecnológicos usados por la NASA y la industria aeroespacial para el diseño de la cámara de combustión del generador. Como un paso preliminar a la construcción de la herramienta, se quiere probar que los gases de combustión producidos por el generador de vapor no tengan un efecto adverso sobre la inyección continua de vapor, sino que por el contrario, éstos sean de ayuda en la eficiencia del proceso.

\section{Generadores de vapor en fondo}

Los generadores de vapor en fondo fueron creados en la década de los setenta/ochenta por empresas como la Sandia National Laboratories, Phillips Petroleum, entre otras, con ánimo de dar solución a ciertas limitaciones que tenían los equipos en superficie [4,7-9].

Dentro de las ventajas más notables que tienen los DSG, se enuncia minimizar pérdidas de energía en el recorrido de superficie y fondo, hasta la cara de la formación, reducir el impacto ambiental ya que los gases de combustión pueden ser inyectados a la formación, disminuyendo la contaminación del aire y economizando gastos invertidos en lavadores que eliminen y controlen $\mathrm{SO}_{x}$ y $\mathrm{NO}_{x}$; otra ventaja valiosa de los generadores de vapor en fondo es su capacidad de operar a profundidades mayores de tres mil pies, permitiendo recuperar un gran volumen de crudo pesado en el mundo. Adicionalmente, los gases generados por la combustión ayudan al mantenimiento de presión del yacimiento, esto es de gran importancia debido a que se requieren altas presiones para que el aceite fluya hasta los pozos productores.

Los DSG pueden usarse en yacimientos offshore que requieren de un proceso térmico para reducir la viscosidad del crudo, puesto que el vapor generado con una máquina en superficie se condensaría muy rápido debido a la columna de agua; además, los equipos convencionales ocupan mayor espacio en la plataforma y necesitan de más facilidades de superficie, razón por la cual un equipo en fondo resulta ser una alternativa atractiva [6,7].

Los generadores de vapor en fondo reciben una clasificación de acuerdo al tipo de energía que usan para generar calor. En la actualidad existen numerosos prototipos y patentes que van desde generadores de combustión hasta generadores que usan energía nuclear o electromagnética. Los primeros son los más comunes y los de interés en el presente artículo. Algunas patentes son: United States Patent Office 3'456.721 Julio 22, 1969. United States Patent 4'237.973 Diciembre 9, 1980. United States Patent 4'390.062 Junio 28, 1983.

\section{Generadores de combustión}

Los generadores de combustión son aquellos que a través de la reacción química de un comburente (oxígeno) y un combustible (propano, aceite in situ, entre otros) generan calor para transformar el agua líquida en vapor. Para su funcionamiento requieren de varias tuberías de alimentación: agua, combustible y aire, razón por la cual son herramientas de gran complejidad en cuanto a su instalación en el pozo y funcionamiento. Éstos a su vez se sub-clasifican en generadores de contacto directo o de alta presión y de contacto indirecto o de baja presión.

Generadores de combustión de contacto directo. Son herramientas tubulares cuyo ensamblaje consta de un atomizador, el cual controla la cantidad de combustible que entra, con el fin de minimizar el tiempo de combustión; un quemador, situado en el extremo superior de la herramienta, donde el aire es debidamente mezclado con las gotas de combustible para generar una reacción de combustión estable que calentará el agua que se inyecta desde las paredes laterales de la cámara y se pone en contacto con los gases y la llama que sale del quemador; un mezclador, donde el agua se combina uniformemente con los productos de la combustión, y un vaporizador, cuya función es proporcionar un tiempo de residencia suficiente para alcanzar la evaporación completa del agua [8]. La característica más representativa de esta clase de generadores es que los gases de combustión se mezclan con el vapor que va a ser inyectado a la formación y salen por el extremo inferior de la herramienta que se encuentra abierto.

\footnotetext{
Generadores de combustión de contacto indirecto. Tienen un funcionamiento similar a los generadores de combustión de contacto directo; su diferencia radica en que los gases de combustión no se mezclan con el vapor, puesto que los primeros retornan a superficie por medio de una tubería.
} 
El generador es alimentado con aire y combustible a través de dos tuberías que llegan a la cámara de combustión, donde luego reaccionarán liberando la energía necesaria para evaporar el agua. El agua ingresa al equipo por una tubería externa a la cámara de combustión, y simultáneamente, gracias al calor de los gases de combustión, ocurre un intercambio de calor de forma que el agua pasa a fase vapor. Finalmente, los gases de combustión salen por una tubería ubicada en la parte superior del generador y llegan a superficie, mientras que el vapor sale por la parte inferior del equipo [6]. En la Figura 1 se ilustran las partes fundamentales de un generador de vapor de contacto directo e indirecto. Los generadores de vapor en fondo presentan ciertas restricciones, entre ellas el diámetro de la herramienta, que está limitado al diámetro del casing de producción. A partir de esto, cada DSG es único y diseñado especialmente para cubrir los requerimientos del pozo en el que se ingresará la herramienta.

Otra condición, aunque más pertinente para el caso de un generador de contacto directo, es que la presión de la unidad debe estar 300psi por encima de la presión de yacimiento, para que los gases de escape penetren en la formación; de igual modo, la potencia de salida de la unidad es un parámetro importante y se recomienda ser de al menos 7MMBTU/h (millones de BTU por hora), para que la estimulación sea efectiva. Este valor se alcanza con cámaras de combustión de turbinas de gas $[7,8]$.

CONTACTO

DIRECTO

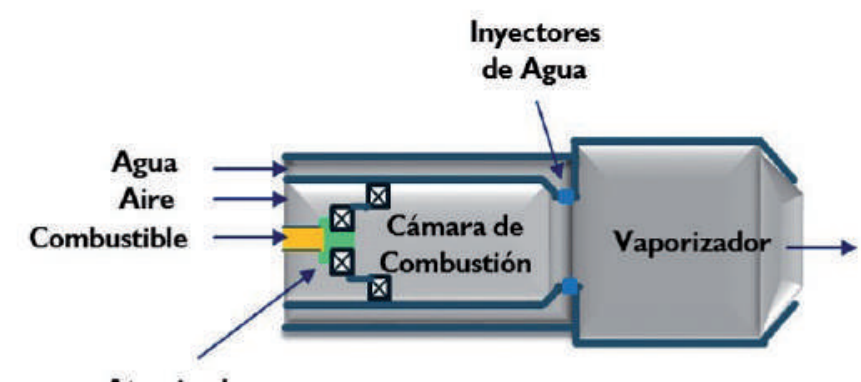

Atomizador

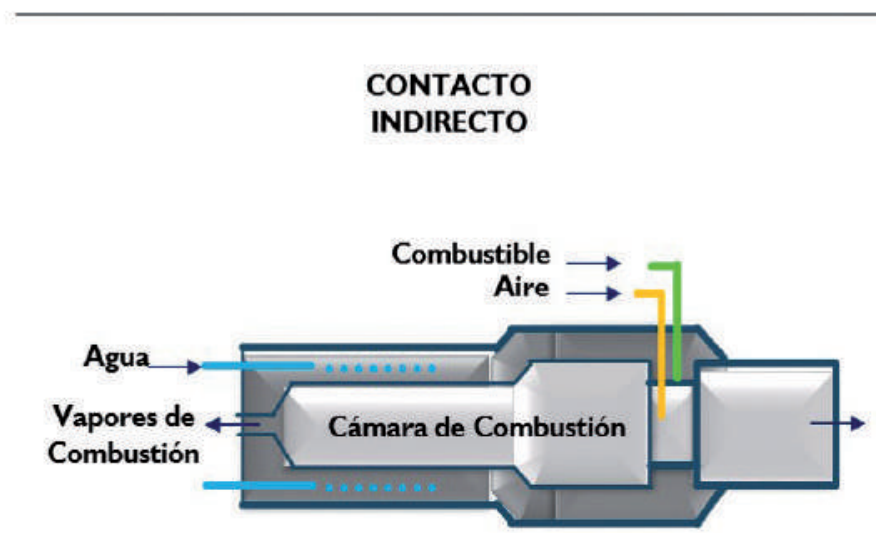

Fuente. Schirmer R., Eson R., "A Direct Fired Downhole Steam Generator - From design to field test”, SPE-Phillips Petroleum, 1985. Nguyen, D., Singh, S., Wong, S., "The commercial viability and comparative economics of downhole steam generators in Alberta.", Alberta Research Council, JCPT, 1988 - Modificado por Autores.

Figura 1. Partes elementales de un DSG de contacto directo e indirecto. 
En diferentes pruebas y pilotos realizados con generadores de vapor en fondo, se han detectado problemas de corrosión en la herramienta y en las líneas, por lo que el mantenimiento y el control en la ejecución del proceso es de orden imperativo $[8,10]$. En la Figura 2, se muestra el posicionamiento de un DSG en el tubing, y algunos requerimientos y recomendaciones que se sugieren en la implementación de la herramienta, como el uso de aislantes y aditivos especiales en la lechada de cemento, puesto que la herramienta se encuentra trabajando a altas temperaturas y la integridad del pozo se puede ver comprometida.

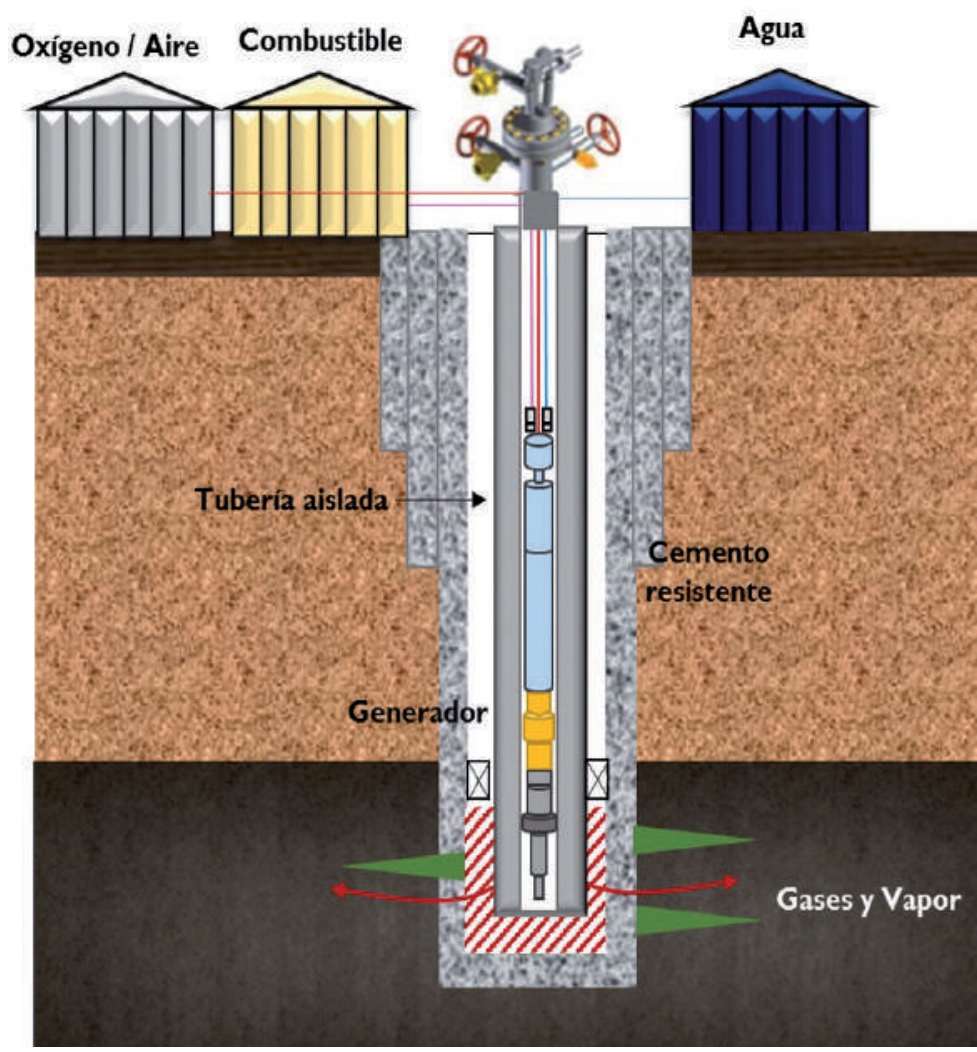

Fuente: Autores

Figura 2. Vista general de un generador de vapor en fondo.

\section{Gases de combustión}

Normalmente, los combustibles que se usen en el generador condicionarán los productos de las reacciones de combustión. Los combustibles más comunes son el diésel y el propano, aunque también se usan combustibles gaseosos más elementales como el metano.

Así mismo, la reacción de combustión dependerá básicamente del diámetro de la herramienta ya que en un diámetro limitado, el oxígeno disminuye, predominando las reacciones de combustión incompletas. Algunos gases que se encuentran a la salida del generador son el dióxido de carbono $\left(\mathrm{CO}_{2}\right)$, nitrógeno $\left(\mathrm{N}_{2}\right)$, monóxido de carbono $(\mathrm{CO})$, oxígeno $\left(\mathrm{O}_{2}\right)$, y vapor de agua $\left(\mathrm{H}_{2} \mathrm{O}\right)$.

De acuerdo al gas predominante, el efecto en el yacimiento será diferente. Cuando se usa un generador de contacto directo, se observa el frente de gases $\left(\mathrm{CO}_{2}\right.$ y $\mathrm{N}_{2}$ fundamentalmente) que se mueve delante del frente de vapor, tal como lo ilustra la Figura 3. Si el dióxido de carbono, alcanza la presión mínima de miscibilidad (PMM) la cual se define como la presión más baja a la cual se puede alcanzar la miscibilidad sea a primer contacto o al multicontacto a temperatura y composición constantes, se disolverá en el aceite reduciendo la viscosidad del mismo, reafirmando la acción del vapor, y mejorando también otras 
propiedades del crudo. Como las fuerzas viscosas son de menor magnitud, por la acción previa del $\mathrm{CO}_{2}$, el frente de vapor será eficiente puesto que no pierde mucha energía en su recorrido y la cantidad de agua condensada será menor. La combinación de vapor y gases de combustión modifica el perfil de inyección, ya que a medida que se disuelve y difunde el $\mathrm{CO}_{2}$ en el crudo, el vapor tendrá un mayor radio de calentamiento. $[3,11,12]$

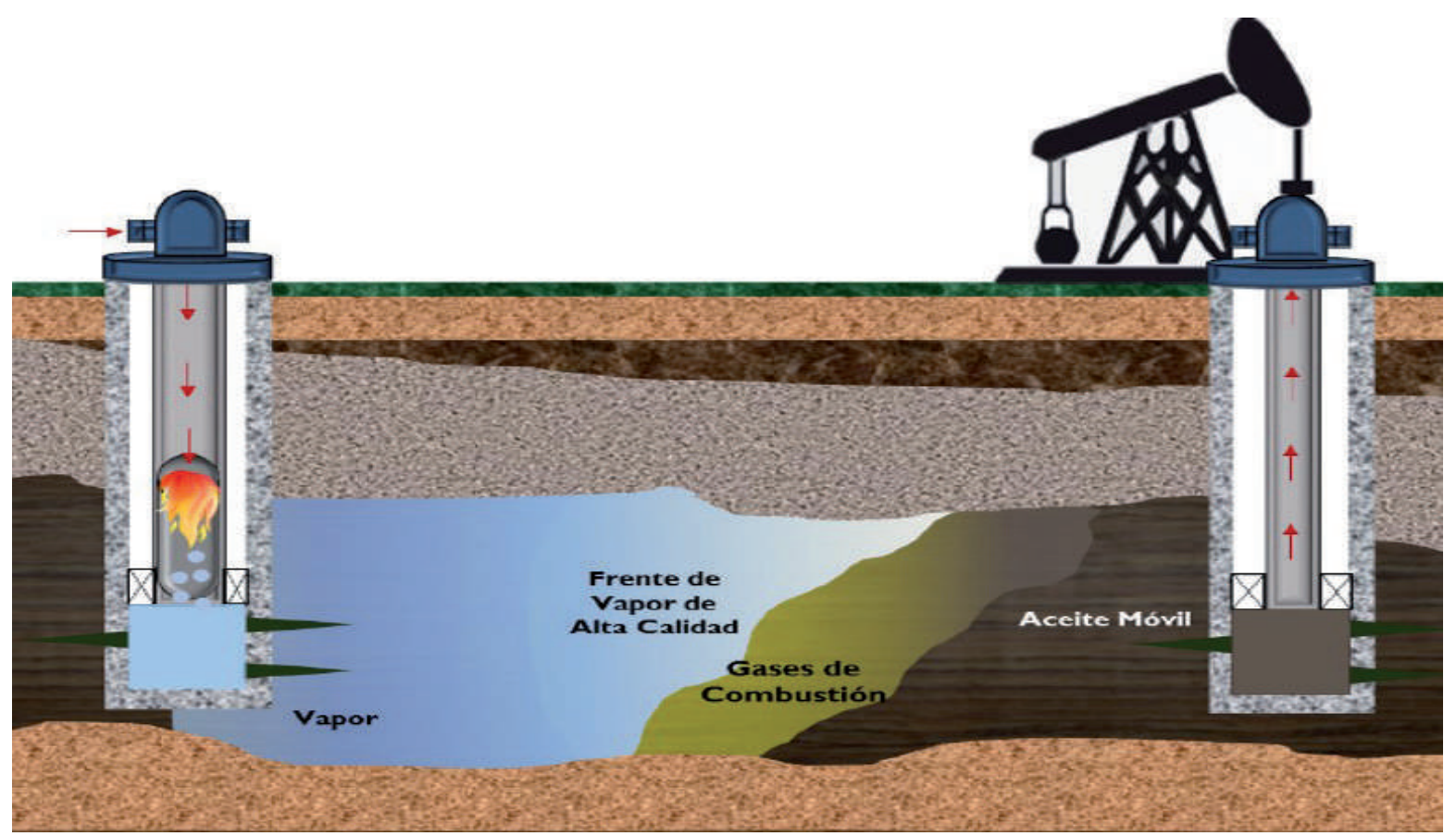

Fuente. Castrogiovanni A., "Benefits and Technical Challenges of Downhole Steam Generation for Enhanced Oil Recovery", SPE, 2011 - Modificado por Autores.

Figura 3. Inyección de vapor con un generador de vapor en fondo de contacto directo.

Si el $\mathrm{CO}_{2}$ no alcanza la PMM se comportará de forma inmiscible, al igual que el $\mathrm{N}_{2}$, que tiene presiones mínimas de miscibilidad elevadas (superiores a los 5.000psi [13]), En condiciones inmiscibles existe recuperación de aceite por hinchazón y vaporización limitada del gas; no obstante, el mecanismo predominante es de desplazamiento por empuje.

\section{Modelo conceptual de simulación}

El presente modelo conceptual se construyó en el software STARS de la compañía CMG ${ }^{\circledR}$ (Computer Modelling Group Ltd.) y se realizó con el fin de comprobar o refutar numerosas hipótesis que se han creado en torno al efecto de los gases producto de la combustión de un DSG.

Para la creación del modelo se escogió un grid cartesiano tridimensional con un tamaño de 10 acres, discretizado en 50 celdas en la dirección i y j, y 18 celdas en la dirección $k$.

El modelo es totalmente homogéneo, y presenta un espesor de arena neto de $90 \mathrm{ft}$. Las propiedades petrofísicas se muestran en la Tabla 1, al igual que las propiedades térmicas de la roca y los fluidos del modelo, ya que se representará un proceso térmico de inyección continua de vapor [14].

En cuanto a las propiedades de los fluidos del yacimiento, se creó un PVT (presión, volumen específico y temperatura) sintético con valores característicos de un aceite negro, las cuales también se muestran en la Tabla 1.

Para modelar correctamente el comportamiento del fluido a valores de presión mayores a la presión de burbuja, se escogieron las correlaciones de Vázquez \& Beggs y Kartoatmodjo \& Schmidt, mostradas en las Ecuaciones 1 y 3 , para corregir la viscosidad del aceite y el factor volumétrico de formación del aceite respectivamente [15]. 
Conociendo el valor de viscosidad a condiciones de yacimiento, $1.000 \mathrm{cP}$, y el valor al cual se querían generar datos, se construyeron las gráficas a partir del punto de burbuja para que éstas fueran funciones continuas.

Tabla 1. Propiedades del Modelo de Simulación.

\begin{tabular}{|c|c|}
\hline Propiedad & Valor \\
\hline Profundidad $<\mathrm{ft}\rangle$ & 2.500 \\
\hline Espesor $<f t>$ & 90 \\
\hline Porosidad $<a d>$ & 0,3 \\
\hline $\mathrm{Kv} / \mathrm{Kh}<\mathrm{ad}>$ & 0,2 \\
\hline Saturación de Agua $<a d>$ & 0,2 \\
\hline Saturación de Aceite <ad > & 0,8 \\
\hline Presión del Yacimiento <psi> & 1.100 \\
\hline Conductividad Térmica $\left\langle B T U / d f t^{\circ} F\right\rangle$ & 44 \\
\hline Capacidad Calorífica $<B T U / f^{3{ }^{\circ}} \mathrm{F}>$ & 36 \\
\hline Compresibilidad $<1 / p s i>$ & $5^{*} 10^{-6}$ \\
\hline $\begin{array}{c}\text { Conductividad Térmica del Aceite } \\
\qquad B T U / d^{\circ} t^{\circ}>\end{array}$ & 2 \\
\hline $\begin{array}{l}\text { Conductividad Térmica del Gas } \\
<B T U / d^{\circ}{ }^{\circ} F>\end{array}$ & 1,2 \\
\hline $\begin{array}{c}\text { Conductividad Térmica del Agua } \\
<B T U / d^{\circ} t^{\circ} F\end{array}$ & 8,6 \\
\hline Temperatura de Yacimiento $<^{\circ} \mathrm{F}>$ & 105 \\
\hline Densidad del Crudo $<^{\circ} \mathrm{APl}>$ & 12 \\
\hline Presión de Saturación <psi> & 100 \\
\hline Gravedad Específica del Gas $\langle a d\rangle$ & 0,6 \\
\hline
\end{tabular}

$$
\mu_{0}=\mu_{\mathrm{b}} \frac{\mathrm{Pm}}{\mathrm{Pb}}
$$

Donde,

$$
\mathrm{m}=2,6 \mathrm{P}^{1,187} \mathrm{e}^{-11,513-\left(8,98^{*} 10-5 \mathrm{P}\right)}
$$

$\mu_{\mathrm{o}}=$ Viscosidad del aceite, $\mathrm{cP}$

$\mu_{\mathrm{b}}=$ Viscosidad del aceite en el punto de burbuja, $\mathrm{CP}$

$\mathrm{P}=$ Presión, psi

$\mathrm{P}_{\mathrm{b}}=$ Presión de burbuja, psi

$$
\mathrm{B}_{\mathrm{o}}=\mathrm{B}_{\mathrm{ob}} \mathrm{e}^{(\mathrm{Co}(\mathrm{Pb}-\mathrm{P}))}
$$

Donde,

$$
\mathrm{B}_{\mathrm{ob}}=0,98496+1 * 10^{-4} \mathrm{~F}^{1.5}
$$

$$
\mathrm{F}=\mathrm{R}_{\mathrm{sb}} 0,755 \Upsilon_{\mathrm{gc}} 0.25 \Upsilon_{\mathrm{o}}{ }^{-1.5}+0,45 \mathrm{~T}
$$

$\mathrm{B}_{\mathrm{ob}}=$ Factor volumétrico de formación del aceite en el punto de burbuja, RB/STB

$B_{0}=$ Factor volumétrico de formación del aceite, $\mathrm{RB} / \mathrm{STB}$

$\mathrm{T}=$ Temperatura, ${ }^{\circ} \mathrm{F}$

$\mathrm{C}_{\mathrm{o}}=$ Compresibilidad del aceite, $\mathrm{psi}^{-1}$

$\mathrm{R}_{\mathrm{sb}}=$ Relación de gas disuelto en aceite, SCF/STB

$\gamma_{\mathrm{gc}}=$ Gravedad específica del gas, Adimensional

$\gamma_{0}=$ Gravedad específica del aceite, ${ }^{\circ} \mathrm{API}$

$\mathrm{P}=$ Presión, psi

$\mathrm{P}_{\mathrm{b}}=$ Presión de burbuja, psi

La construcción de las curvas de permeabilidad relativa se realizó con end points a través de la correlación de Hirasaki, mostrada en las Ecuaciones 6 y 7 . En la Figura 4 se muestra la gráfica de permeabilidad relativa agua - aceite, la cual describirá el movimiento de los fluidos en el medio poroso y en la que se distingue un sistema mojado por agua. Para la configuración de los pozos, se utilizó un arreglo de cinco puntos invertidos, como se muestra en la Figura 5, que encierra un área de 5 acres. El constraint usado para este tipo de pozos fue una BHP (bottom hole pressure) de 200psi.

$$
\begin{gathered}
\mathrm{k}_{\mathrm{rw}}=\mathrm{k}_{\mathrm{rw}(\text { Sor })} \mathrm{S}_{\mathrm{w}}{ }^{*} \mathrm{Nw} \\
\mathrm{k}_{\mathrm{ro}}=\mathrm{k}_{\mathrm{ro} \text { (Swirr) }}\left(1-\mathrm{S}_{\mathrm{w}}{ }^{*}\right)^{\mathrm{No}}
\end{gathered}
$$

Donde,

$$
\mathrm{S}_{\mathrm{w}}{ }^{*}=\frac{\mathrm{S}_{\mathrm{w}}-\mathrm{S}_{\mathrm{wc}}}{1-\mathrm{S}_{\mathrm{or}-\mathrm{S}_{\mathrm{wc}}}}
$$

$\mathrm{Kr}_{\mathrm{w}}=$ Permeabilidad relativa del agua, $\mathrm{mD}$

$\mathrm{Kr}_{\mathrm{o}}=$ Permeabilidad relativa del aceite, $\mathrm{mD}$

$\mathrm{Kr}_{\mathrm{w} \text { (Sor) }}^{\circ}=$ Permeabilidad relativa del agua a condiciones de saturación de aceite residual, $\mathrm{mD}$ $\mathrm{Kr}_{\text {o(Swirr) }}=$ Permeabilidad relativa del aceite a condiciones de saturación de agua irreducible, $\mathrm{mD}$

$\mathrm{S}_{\mathrm{w}}=$ Saturación de agua, adimensional

$S_{w c}=$ Saturación de agua crítica, adimensional $\mathrm{S}_{\mathrm{or}}=$ Saturación de aceite residual, adimensional $\mathrm{N}_{\mathrm{o}}, \mathrm{N}_{\mathrm{w}}=$ exponentes de Hirasaki 


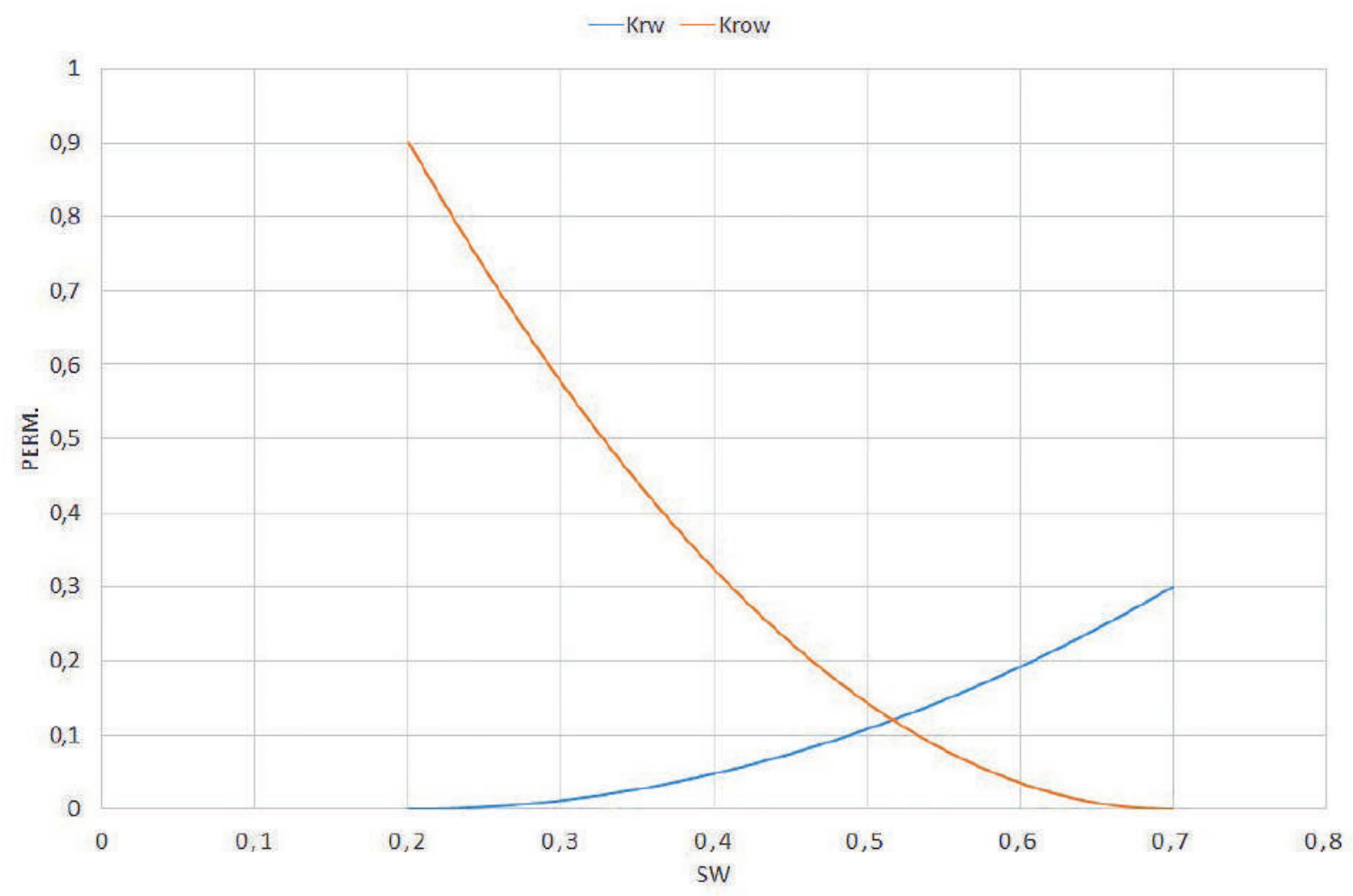

Figura 4. Permeabilidad relativa agua - aceite.

Con el fin de tener resultados valederos, previo a la inyección de vapor, se debe ajustar el modelo de simulación. La producción primaria del yacimiento se simuló por 3 años, desde el 2015 hasta el 2018, debido a que el modelo presentaba una rápida caída de presión, característica propia de un aceite pesado. Adicionalmente, como reflejo de la baja cantidad de aceite producido se obtuvo un factor de recobro bajo, de aproximadamente $3,3 \%$, un valor lógico cuando el único mecanismo de producción es la expansión de roca y los fluidos.

Para la simulación del proceso térmico, se plantearon tres escenarios: el primero fue la implementación de un proceso de inyección continua de vapor con un generador en superficie; debido a que la profundidad del yacimiento es de $2.500 \mathrm{ft}$, la calidad del vapor usada fue de $60 \%$. En el segundo caso se aumentó la calidad del vapor hasta un valor de $85 \%$, dato que puede ser logrado con un generador de vapor en fondo de contacto indirecto. Finalmente, el tercer caso simulado fue el DSG de contacto directo (calidad de vapor $85 \%$ más los gases de salida producidos en la combustión del generador); la proporción de los gases de salida del generador, y por tanto entrada a la formación fueron: Vapor $62 \%$, Nitrógeno $32 \%$, Dióxido de Carbono 5\%, Oxígeno 1\% [7].

En los tres casos se usó una temperatura de inyección de $590^{\circ} \mathrm{F}$ acorde con la presión de inyección de 1.300psi; adicionalmente, la tasa de inyección escogida fue de $675 \mathrm{bbl} / \mathrm{d}$ [1].

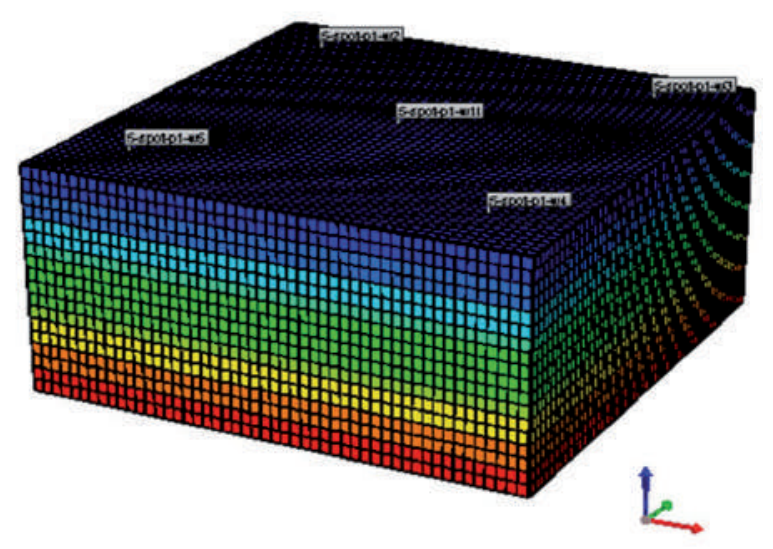

Figura 5. Modelo de Simulación: posición de los pozos. 


\section{Resultados de la simulación}

A continuación, se muestran los resultados comparativos de los tres casos simulados, en cuanto producción de aceite, factor de recobro y efecto de los gases de combustión.

En la gráfica ilustrada en la Figura 6, se aprecia un aumento considerable en la producción de aceite, cuando se usa un DSG de contacto directo, con una respuesta casi inmediata, comprobando que sí hay un efecto favorable de los gases de combustión sobre el aceite in situ.

La Figura 7 muestra las curvas del factor de recobro, relacionadas directamente con las curvas de aceite producido; en éstas se puede apreciar que no hay una diferencia representativa entre la curva de un DSG de contacto indirecto con una calidad de $85 \%$, y un generador ubicado en superficie con calidad de $60 \%$; si el modelo conceptual tuviese una profundidad mayor a la limitante en un proceso de vapor, habría una diferencia más significativa debido a las pérdidas de calor en pozo; el mayor factor de recobro se alcanza con un DSG de contacto directo, llegando a valores de $63 \%$. A pesar de que el gas no condensable que se encontraba en mayor proporción era el nitrógeno, caracterizado por sus altos valores de PMM, se graficó el cambio de la viscosidad del aceite para el caso de un generador de vapor de contacto directo con comburente aire, y un generador de vapor de contacto indirecto, con el fin de determinar la existencia de miscibilidad gracias al cambio de viscosidad y corroborar el efecto de los gases de combustión.

Se encontró que sí hubo miscibilidad entre los gases de combustión del generador de vapor en fondo y el aceite de yacimiento; estos gases además tienen un efecto benéfico, ya que controlan el avance del frente de vapor como se muestra en las Figuras 8 y 9 , en la capa superior del modelo.

Para un tiempo posterior, el conjunto gases - vapor, se ha propagado de forma más eficiente que el caso comparativo de sólo vapor, como se ilustra en las Figuras 10 y 11, corroborando que, por la acción de los gases, el frente de vapor es más estable y tiene una mejor eficiencia de barrido areal $[12,16,17]$.

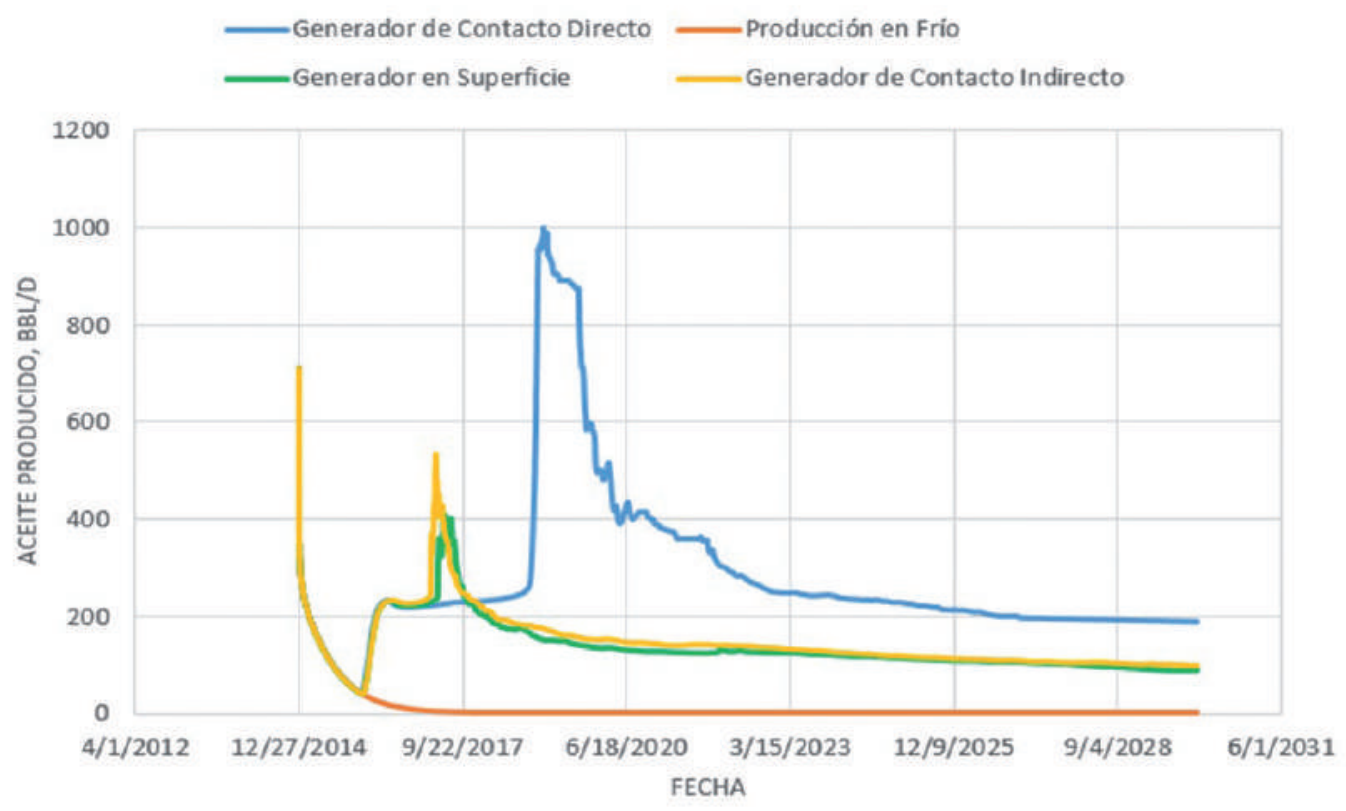

Figura 6. Producción de aceite contra tiempo. 


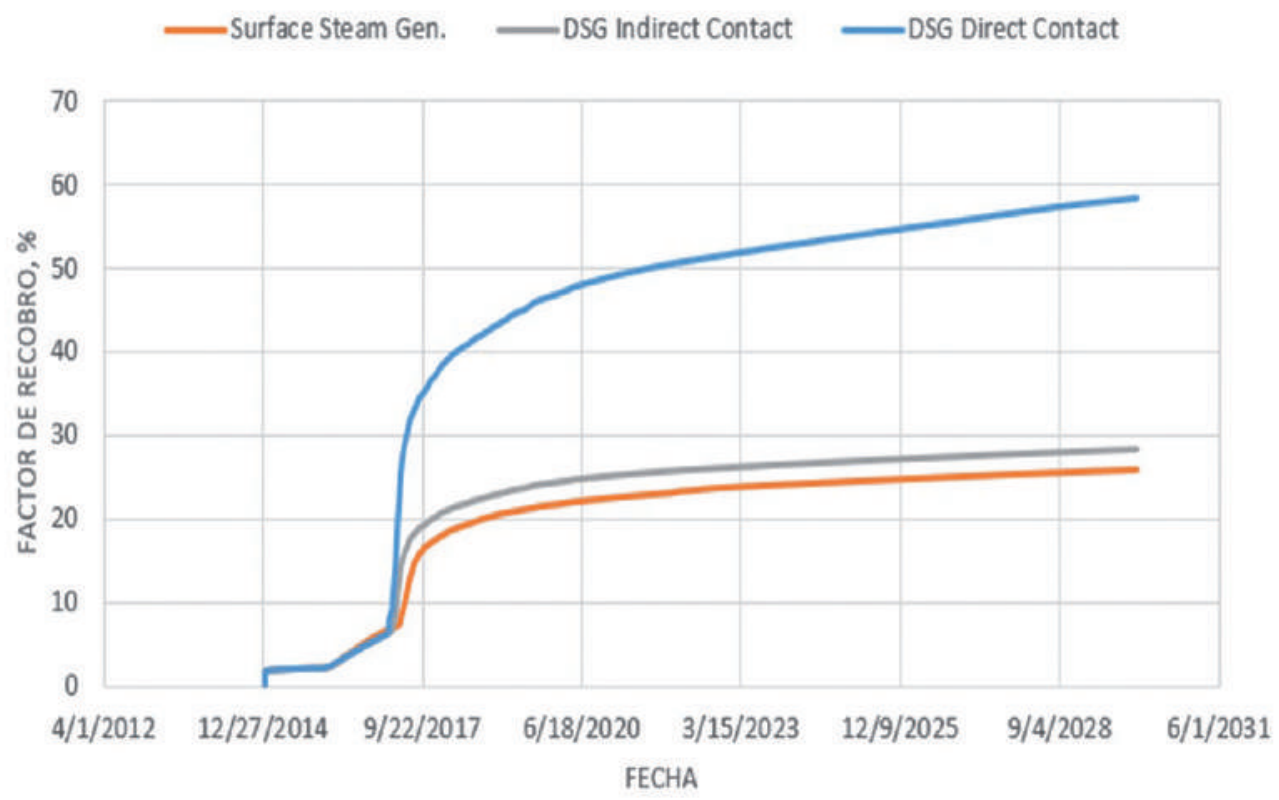

Figura 7. Factor de recobro de aceite contra tiempo.

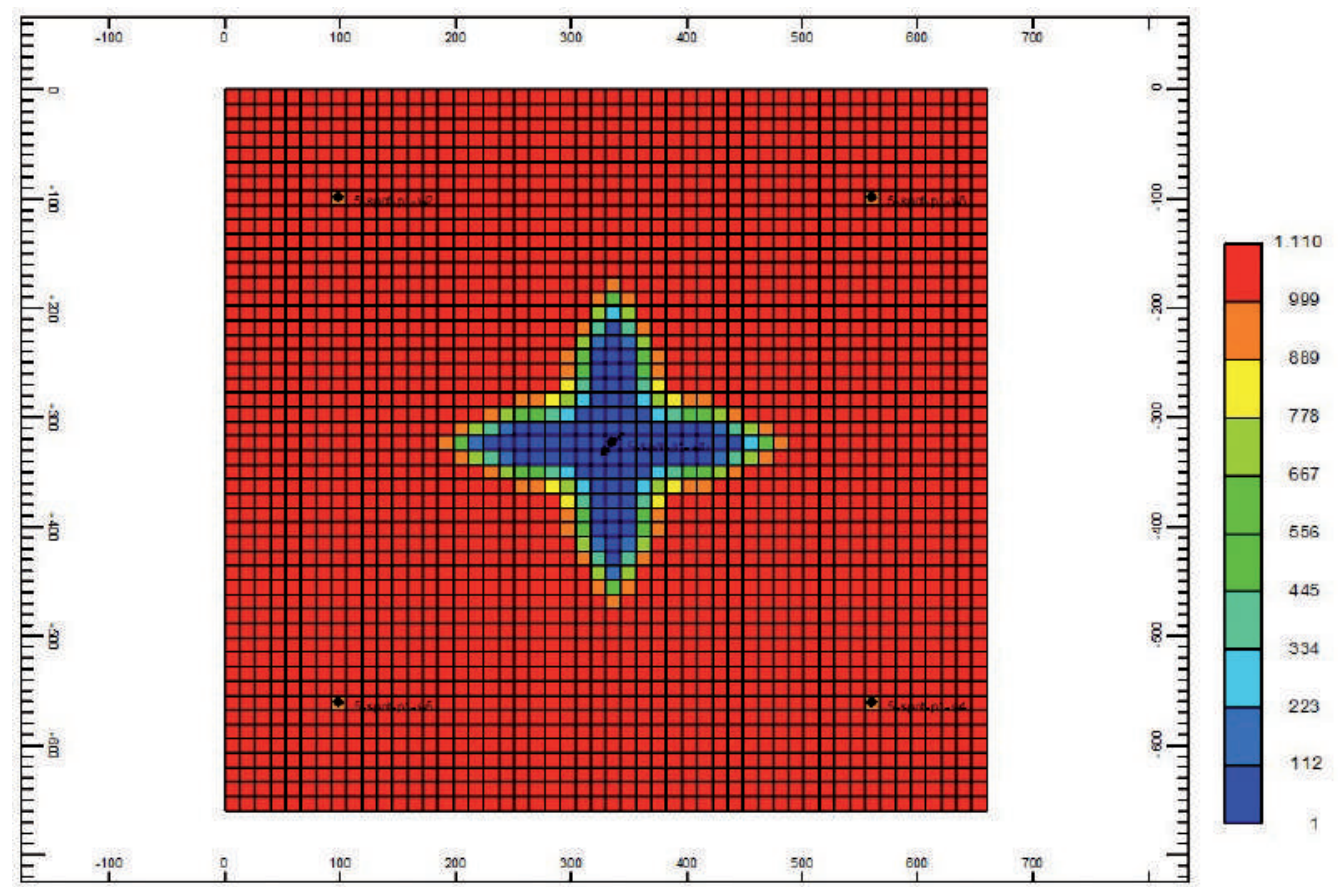

Figura 8. Viscosidad del crudo con un generador de contacto directo: tiempo 2016 -07-01. 


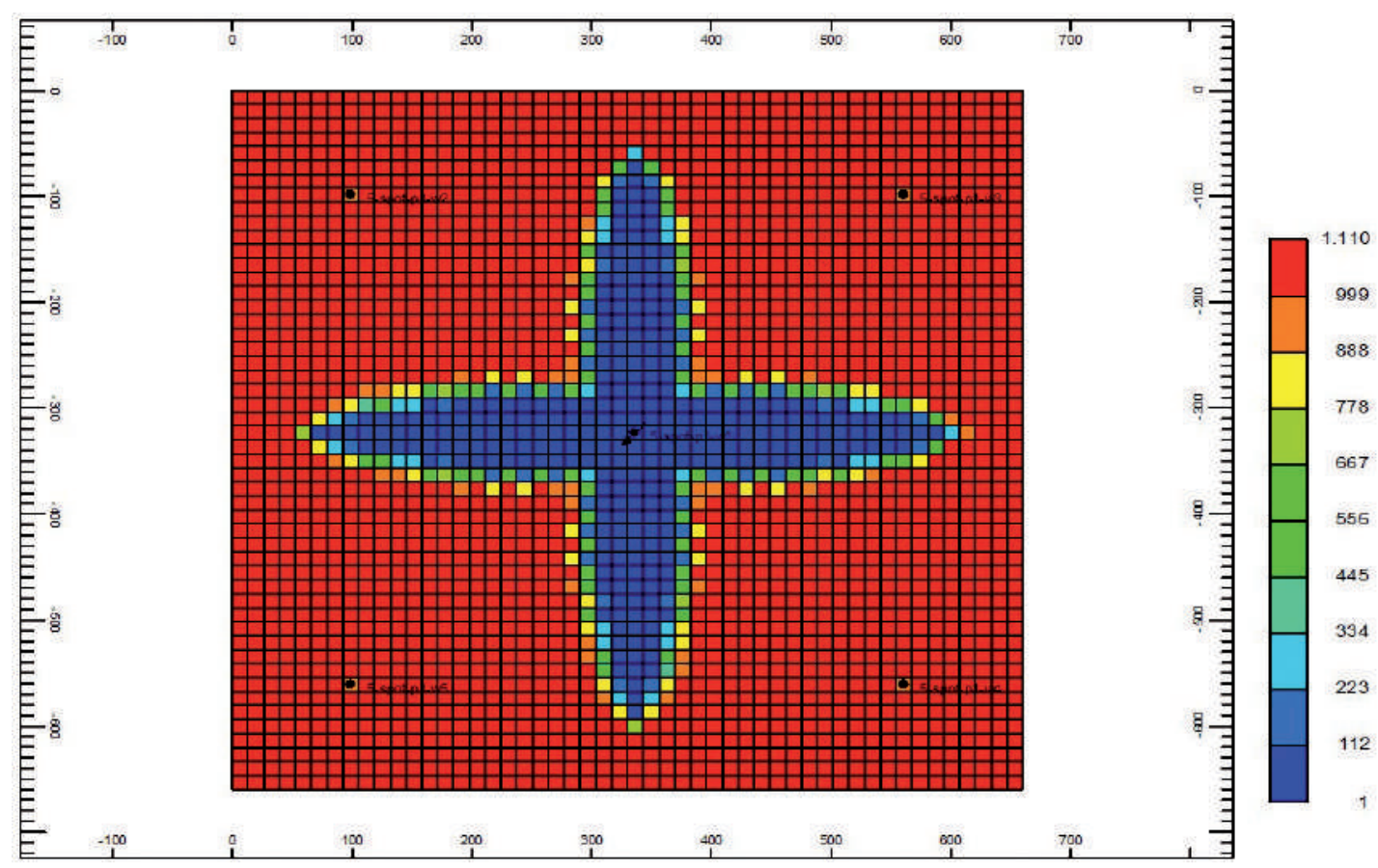

Figura 9. Viscosidad del crudo con un generador de contacto indirecto: tiempo 2016 -07-01.

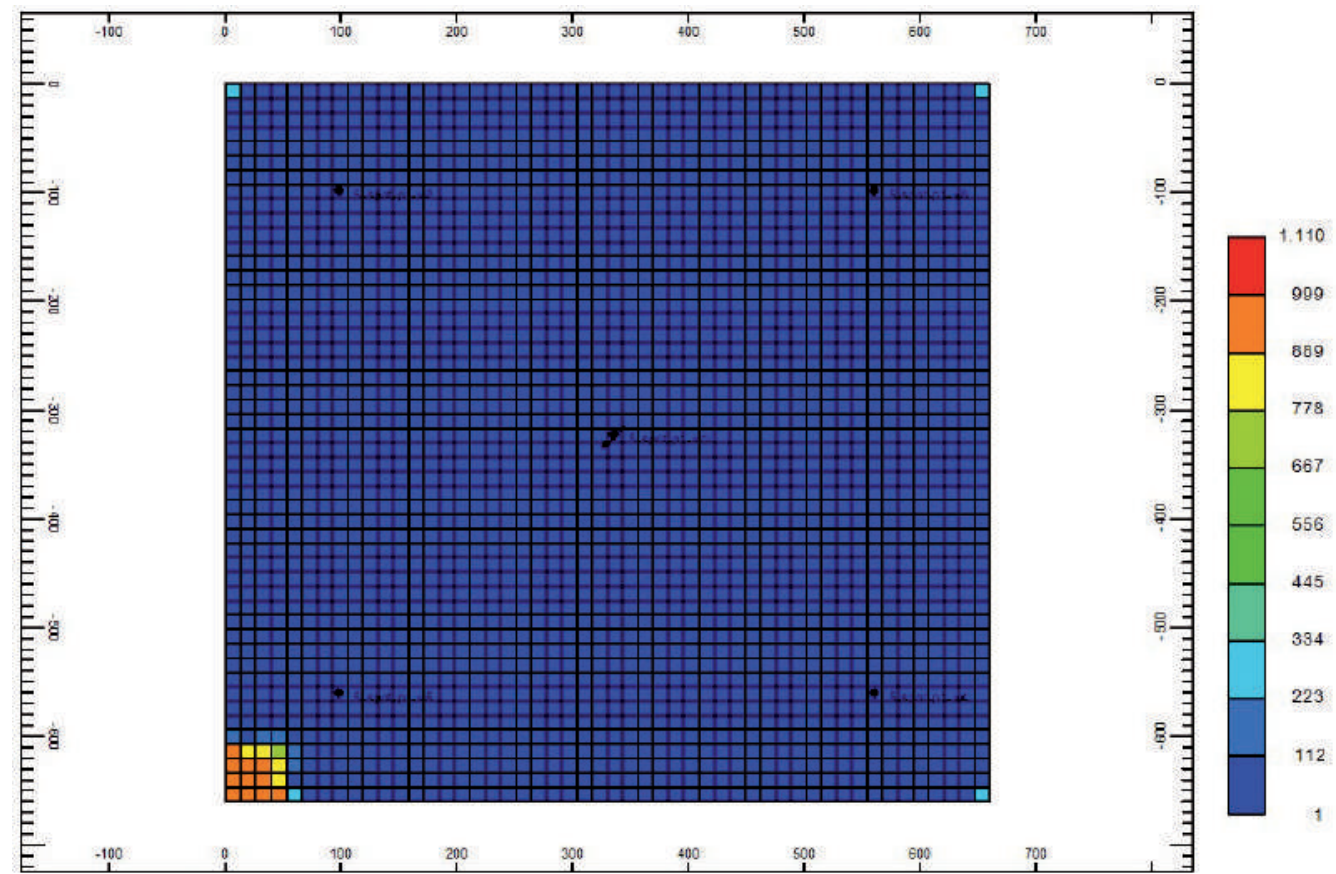

Figura 10. Viscosidad del crudo con un generador de contacto directo: tiempo 2022 -01-01. 


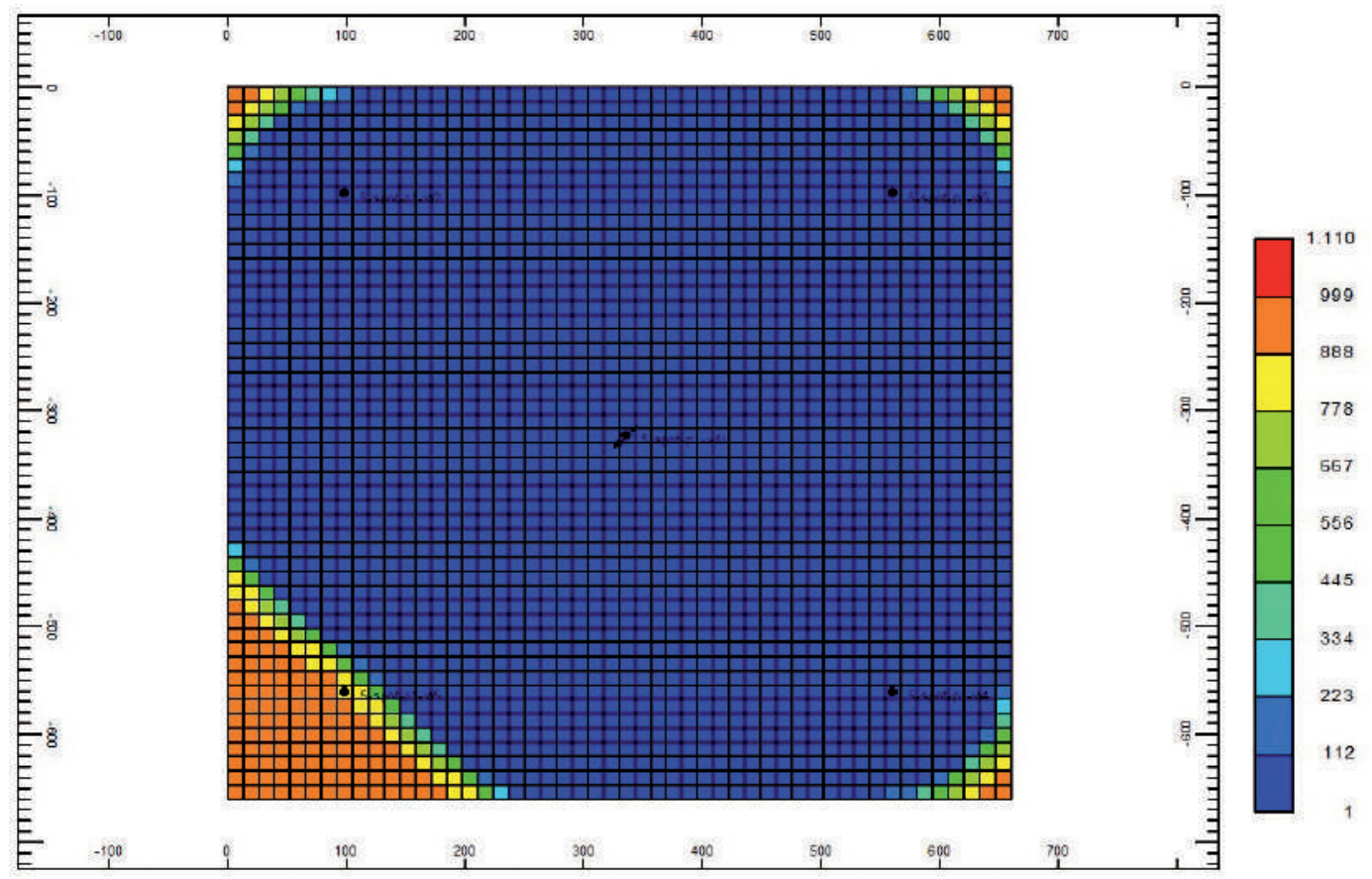

Figura 11. Viscosidad del crudo con un generador de contacto indirecto: tiempo 2022 -01-01.

\section{Conclusiones}

En el estudio de simulación realizado se concluyó que el efecto que tienen los gases producto de la combustión de un generador en fondo en la recuperación de aceite es favorable, y que la inyección continua de vapor se puede optimizar mediante el uso de un DSG, porque éste inyecta gases como el $\mathrm{CO}_{2}$ y el $\mathrm{N}_{2}$, que permiten que el frente de vapor sea más eficiente. Con base en estos estudios se abre una serie de oportunidades de investigación como el diseño de un generador de vapor en fondo de pozo, la adecuación del pozo para dicho proceso y la evaluación técnica y económica de su implementación.

\section{Factores de conversión}

\begin{tabular}{c}
\hline Sistema Métrico \\
\hline Bbl $\times 1,589873 \mathrm{E}-01=\mathrm{m}^{3}$ \\
$\mathrm{BTU} \times 1,055056 \mathrm{E}+00=\mathrm{kJ}$ \\
${ }^{\circ} \mathrm{F} \quad\left({ }^{\circ} \mathrm{F}-32\right) / 1,8={ }^{\circ} \mathrm{C}$ \\
$\mathrm{ft} \times 3,048 \mathrm{E}-01=\mathrm{m}$ \\
in $\times 2,54 \mathrm{E}+00=\mathrm{cm}$ \\
$\mathrm{lbm} \times 4,535924 \mathrm{E}-01=\mathrm{kg}$ \\
$\mathrm{psi} \times 6,894757 \mathrm{E}-03=\mathrm{MPa}$ \\
$\mathrm{Bbl} \times 1,589873 \mathrm{E}-01=\mathrm{m}^{3}$ \\
BTU $\times 1,055056 \mathrm{E}+00=\mathrm{kJ}$
\end{tabular}

\section{Referencias bibliográficas}

[1] Donaldson EC, Chilingarian GV, Yen TF. Screening criteria for steamflood prospects. In: Enhanced Oil Recovery II- Processes and Operations. Países Bajos: Elsevier; 1989.

[2] Agencia Nacional de Hidrocarburos - ANH. Cuenca Llanos Orientales Integración Geológica de la Digitalización y Análisis de Núcleos. Colombia: Agencia Nacional de Hidrocarburos; 2012.

[3] Castrogiovanni A, Fitzpatrick AN, Ware $\mathrm{CH}$. Benefits and technical challenges of downhole steam generation for enhanced oil recovery. In: Canadian Unconventional Resources Conference; 2011 Nov 15-17; Calgary, Canada. Calgary: Society of Petroleum Engineers; 2011 p. 1-11.

[4] Fox RL, Donaldson AB, MulacAJ. Development of technology for downhole steam production. In: SPE/DOE Enhanced Oil Recovery Symposium; 1981 Apr 5-8; Tulsa, United States. Tulsa: Society of Petroleum Engineers; 1981. p. 65-70.

[5] Hart M. Hart C. Comparative evaluation of surface and downhole steam-generation techniques. In: SPE/DOE Enhanced Oil Recovery Symposium; 1982 Apr 4-7; Tulsa, United States. Tulsa: Society of Petroleum 
Engineers; 1982. p. 417-24.

[6] Schirmer RM, Eson RL. A direct-fired downhole steam generator-from design to field test. J. of petroleum tech. 1985;37(10):1-903.

[7] Marshall BW. Field test of two high-pressure, direct-contact downhole steam generators. Volume I. Air/diesel system. United States: Sandia National Labs; 1983.

[8] Eson RL. Downhole Steam Generator-Field Tests. SPE California Regional Meeting. In: SPE/DOE Enhanced Oil Recovery Symposium; 1982 Mar 24-26; San Francisco, United States. Tulsa: Society of Petroleum Engineers; 1982. p. 301-8.

[9] Singh S, Nguyen D, Wong S. The commercial viability and comparative economics of downhole steam generators in Alberta. J. of Canadian Petroleum Tech. 1988;27(5):67-74.

[10] Marshall BW. Operational experiences of a downhole steam generator. In: SPE California Regional Meeting; 1982 Mar 24-26; California, United States. California: Society of Petroleum Engineers; 1982.

[11] Capper L, Kuhlman MI, Vassilellis GD, Schindler M, Fitzpatrick N. Advancing thermal and carbon dioxide recovery methods beyond their conventional limits: downhole innovation. In: SPE Heavy Oil Conference and Exhibition;
2011 Dec 12-14; Kuwait City, Kuwait. Kuwait City: Society of Petroleum Engineers; 2011. p. 1-24.

[12] Jones J, Capper L, Kuhlman M, Vassilellis G, Schneider M J, Fitzpatrick N. Discussion/ Reply: Downhole Steam Generation Pushes Recovery Beyond Conventional Limits. Journal of Petroleum Technology. 2012;64(09):132-6.

[13] Guerrero C, Zamora H. Evaluación de un proceso de estimulación con $\mathrm{CO}_{2}$ miscible utilizando estimulación numérica (tesis pregrado). Bucaramanga, Colombia: Universidad Industrial de Santander; 2011.

[14] PintoJ, MuñozS. Desarrollo de una metodología para la implementación de la administración de energía en procesos de inyección continua de vapor a través de la simulación numérica de yacimientos y métodos analíticos (tesis pregrado). Bucaramanga, Colombia: Universidad Industrial de Santander; 2013.

[15]Bánzer C. Correlaciones numéricas P.V.T. Venezuela: Universidad de Zulia; 1996.

[16]Anjum S. A techno-economic evaluation of downhole steam generation in enhanced oil recovery. Canada: Dalhousie University; 2005.

[17]Donaldson AB. Reflections on a Downhole Steam Generator Program. United States: Society of Petroleum Engineers; 1997. 